\title{
A Kaluza Klein Treatment of a Graviton and Deceleration Parameter $Q(Z)$ as an Alternative to Standard DE and Its Possible Link to Standard DE Equation of State as Given by Li, Li, Wang and Wang, in 2017
}

\author{
Andrew Walcott Beckwith \\ Physics Department, Chongqing University, Chongqing, China \\ Email: Rwill9955b@gmail.com
}

\begin{abstract}
How to cite this paper: Beckwith, A.W. (2019) A Kaluza Klein Treatment of a Graviton and Deceleration Parameter $\mathrm{Q}(\mathrm{Z})$ as an Alternative to Standard DE and Its Possible Link to Standard DE Equation of State as Given by Li, Li, Wang and Wang, in 2017. Journal of High Energy Physics, Gravitation and Cosmology, 5, 208-217. https://doi.org/10.4236/jhepgc.2019.51012
\end{abstract}

Received: November 28, 2018

Accepted: January 6, 2019

Published: January 9, 2019

Copyright $\odot 2019$ by author(s) and Scientific Research Publishing Inc. This work is licensed under the Creative Commons Attribution International License (CC BY 4.0).

http://creativecommons.org/licenses/by/4.0/

Open Access

\begin{abstract}
First of all, we will reiterate a mechanism for relic early universe gravitons. This is the precursor of the main result of this paper and involves the decay of micro black holes, with relic graviton production. For the $2^{\text {nd }}$ part of graviton physics, we invoke reacceleration of the universe. The case for a four-dimensional graviton mass (non-zero) influencing reacceleration of the universe in five dimensions is stated, with particular emphasis on whether five-dimensional geometries as given below give us new physical insights as to cosmological evolution. The final question is, can DM/DE be explained by a Kaluza Klein particle construction? i.e., the author presents a Kaluza Klein particle representation of a graviton mass with the first term to the right equal to a DM contribution and with the $2^{\text {nd }}$ term to the right being effective DE. This is the $2^{\text {nd }}$ era of DE production. Finally, we in the third part invoke the methodology of a modification of $1 / \mathrm{r}$ potentials, and the reacceleration of the universe as a bridge between the first and second parts of this document, in terms of the physics of DE and gravitons. This is compared with an equation of state given as a confirming basis for perhaps rationalizing a linkage between early universe production of gravitons, and then a subsequent Equation of state, for DE which may be able to predict reasons for quintessence.
\end{abstract}

\section{Keywords}

“Massive” Gravitons, Entropy, Kaluza Klein, Dark Matter, Dark Energy 


\section{Plan of Presentation}

$1^{\text {st }}$ part briefly refers to production of $\mathrm{DE}$ due to as an example graviton release from 100 or more black holes, due to relic conditions: (source for this paper is provided). It is already accepted by JHEPGC and is summed up in [1].

$2^{\text {nd }}$ part refers to $\mathrm{DE}$ as created by Gravitons creating re acceleration of the Universe one billion years Ago (jerk calculation). This is also an accepted JHEPGC paper which will be published later [2].

$3^{\text {rd }}$ part is a modification of gravity which may produce DE. This involves modification of $1 / r$ potentials for the reason brought up in this document. This is also an accepted JHEPGC paper, which will be published later [3].

The conclusion of this document compares both part 1 and part 2, while asking if there exists a bridge between the initial production of gravitons, possibly by the mechanism (Black hole in a micro scale, leading to their rapid decay and delivery of subsequent gravitons) brought up in the $1^{\text {st }}$ part, with the subsequent asking of pertinent links to the reinflation era, which is $z=0.43$, i.e. one billion years ago. This is also compared to a result given by [4], as to reinflation, which may be the bridge between the first and second parts of graviton production. i.e. it is the authors consideration that reconciling the [3] bridge between the $1^{\text {st }}$ and the $2^{\text {nd }}$ part mechanisms may entail answering and fine tuning the details given in part 3, which will be attempted later.

\section{2. $1^{\text {st }}$ Part: Mini Black Holes Decaying, and Graviton Production}

i.e. look at what is given in [1], in its conclusion which we will cite here. i.e. the idea is based upon the formation of a finite number of black holes, which decay. Quoting [1] we have that we will be looking at the following.

Quote,

In Corda's recent work [5], we have a so-called Horizon volume, where $\mathrm{n}$ is the so called quantum number $n$ put in where Planck mass is normalized to 1 , so then, if there are $10^{2}$ black holes of mass $10^{2}$ times Planck mass (will set Planck mass, as (1)

$$
\begin{aligned}
& M \approx 10^{2} m_{\text {Planck }} \\
& \left.\Delta V_{n-1 \rightarrow n}\right|_{\text {Total }} \approx 10^{2} \times \Delta V_{n-1 \rightarrow n} \\
& \approx 10^{2} \times 16 \pi\left|\sqrt{\sqrt{\left(10^{2} m_{\text {Planck }}\right)^{2}-\frac{n}{2}}-\sqrt{\left(10^{2} m_{\text {Planck }}\right)^{2}-\frac{n-1}{2}}}\right|
\end{aligned}
$$

Here we make the following simplification of Equation (47) to read as

$$
\left.\Delta V_{n-1 \rightarrow n}\right|_{\text {Total }} \approx 10^{2} \times \Delta V_{n-1 \rightarrow n} \approx 10^{3} \times 16 \pi \cdot\left(\frac{n}{4 \times 10^{4}}\right) \cdot\left|\sqrt{1-\left(\frac{n-1}{n}\right)}\right|
$$

Our supposition is that there are $10^{2}$ mini black holes, and a mass of $10^{2}$ times Planck mass, per each black hole, so that we perform the following normalization, i.e. find $\mathrm{n}$ for quantum number, so that to first approximation 


$$
16 \pi \cdot\left(\frac{n}{4 \times 10^{4}}\right) \cdot\left|\sqrt{1-\left(\frac{n-1}{n}\right)}\right| \approx 10^{6}
$$

i.e. that say 1000 times Planck length, we have the beginning of say creation of 100 mini black holes, each of mass about 100 times Planck mass which would put a huge restriction upon the admissible value, $n$, whereas giving a quantum value, $n$, for the enhanced quantum perturbation, used for penetration of the initial quantum state so assumed in this document as we go from Pre Planckian to Planckian physics, by emergent field construction. Equation (3) could be used to ascertain a quantum value, $n$, which would be for quantization level used to penetrate beyond the shell used to create the cosmological constant

End of quote.

What we have, is in Equation (3) the initial production of say $10^{6}$ relic gravitons, and we are specifying here that relic graviton production is due, maybe even before the electroweak regime of spacetime, and that this may serve as a template as to initial relic graviton production. If we have graviton production this way, we do have a way to ascertain if we have a new candidate to form DE, as we will state next.

\section{3. $2^{\text {nd }}$ Part: Gravitons with a Non Zero Rest Mass. The KK Treatment}

Consider if there is then also a small graviton mass, i.e., as stated by Beckwith [6] [7]:

$$
m_{n}(\text { Graviton })=\sqrt{\frac{n^{2}}{L^{2}}+\left(m_{\text {graviton rest mass }}=10^{-65} \text { grams }\right)^{2}}=\frac{n}{L}+10^{-65} \text { grams }
$$

Note that Rubakov (2002) works with KK gravitons, without the tiny mass term for a 4 dimensional rest mass included in Equation (7). To obtain the KK graviton/DM candidate representation along RS dS brane world, Rubakov obtains his values for graviton mass and graviton physical states in space-time after using the following normalization: $\int \frac{\mathrm{d} z}{a(z)} \cdot\left[h_{m}(z) \cdot h_{\tilde{m}}(z)\right] \equiv \delta(m-\tilde{m})$. Rubakov [8] (2002) uses $J_{1}, J_{2}, N_{1}, N_{2}$ which are different forms of Bessel functions. His representation of a graviton state is given by Equation (4), which is almost completely acceptable for our problem, since the rest mass of a graviton in four dimensions is so small. If so, then the wave function for a graviton with a tiny 4 dimensional space time rest mass can be written as [8].

$$
h_{m}(z)=\sqrt{m / k} \cdot \frac{J_{1}(m / k) \cdot N_{2}([m / k] \cdot \exp (k \cdot z))-N_{1}(m / k) \cdot J_{2}([m / k] \cdot \exp (k \cdot z))}{\sqrt{\left[J_{1}(m / k)\right]^{2}+\left[N_{1}(m / k)\right]^{2}}}
$$

Equation (5) is for $\mathrm{KK}$ gravitons having a TeV magnitude mass $M_{Z} \sim k$ (i.e., for mass values at $0.5 \mathrm{TeV}$ to above $1 \mathrm{TeV}$ ) on a negative tension RS brane. It would be useful to relate this KK graviton, which is moving with a speed propor- 
tional to $H^{-1}$ with regards to the negative tension brane with $h \equiv h_{m}(z \rightarrow 0)=$ const $\cdot \sqrt{\frac{m}{k}}$ as an initial starting value for the KK graviton mass. If Equation (5) is for a "massive" graviton with a small 4 dimensional graviton rest mass and if $h \equiv h_{m}(z \rightarrow 0)=$ const $\cdot \sqrt{\frac{m}{k}}$ represents an initial state, then one may relate the mass of the KK graviton moving at high speed with the initial rest mass of the graviton. This rest mass of a graviton is

$m_{\text {graviton }}(4$-Dim GR $) \sim 10^{-48} \mathrm{eV}$, opposed to $M_{X} \sim M_{\text {KK Graviton }} \sim 0.5 \times 10^{9} \mathrm{eV}$. Whatever the range of the graviton mass, it may be a way to make sense of what was presented by Dubovsky et al. [9], who argue for a graviton mass, using CMBR measurements, of $M_{\mathrm{KK} \text { Graviton }} \sim 10^{-20} \mathrm{eV}$. Also, Equation (6) will be the starting point used for a KK tower version of Equation (6). So from Maartens [10],

$$
\dot{a}^{2}=\left[\left(\frac{\tilde{\kappa}^{2}}{3}\left[\rho+\frac{\rho^{2}}{2 \lambda}\right]\right) a^{2}+\frac{\Lambda \cdot a^{2}}{3}+\frac{m}{a^{2}}-K\right]
$$

Maartens [12] also gives a $2^{\text {nd }}$ Friedman equation:.

$$
\dot{H}^{2}=\left[-\left(\frac{\tilde{\kappa}^{2}}{2} \cdot[p+\rho] \cdot\left[1+\frac{\rho^{2}}{\lambda}\right]\right)+\frac{\Lambda \cdot a^{2}}{3}-2 \frac{m}{a^{4}}+\frac{K}{a^{2}}\right]
$$

Also, an observer is in the low redshift regime for cosmology, for which $\rho \cong-P$, for red-shift values z from zero to $1.0-1.5$. One obtains exact equality, $\rho=-P$, for $z$ between zero to 0.5 . The net effect will be to obtain, based on Equation (7), assuming $\Lambda=0=K$ and using $a \equiv\left[a_{0}=1\right] /(1+z)$ to get a deceleration parameter $\mathrm{q}$ as given in Equation (8).

$$
q=-\frac{\ddot{a} a}{\dot{a}^{2}}=-1+\frac{2}{1+\tilde{\kappa}^{2}[\rho / m] \cdot(1+z)^{4} \cdot(1+\rho / 2 \lambda)} \approx-1+\frac{2}{2+\delta(z)}
$$

These set values, allow a graviton-based substitute for DE. $\Lambda=0=K$ plus a small rest mass for a graviton in four dimensions allows for "massive gravitons" that behave the same as DE. Setting $\Lambda=0=K$, while having a modified behavior for the density expression, for a Friedman equation with small 4 dimensional graviton mass, means that dark energy is being replaced by a small 4 dimensional rest mass for a graviton.

\section{$2^{\text {nd }}$ part, continued. Consequences of small graviton mass for reaccelera- tion of the universe}

In a revision of Alves et. al. [11], Beckwith [6] [7] used a higher-dimensional model of the brane world combined with KK graviton towers per Maartens [10]. The energy density $\rho$ of the brane world in the Friedman equation is used in a form similar to Alves et al. [11] by Beckwith [6] [7] for a non-zero graviton:

$$
\rho \equiv \rho_{0} \cdot(1+z)^{3}-\left[\frac{m_{g} \cdot(c=1)^{6}}{8 \pi G(\hbar=1)^{2}}\right] \cdot\left(\frac{1}{14 \cdot(1+z)^{3}}+\frac{2}{5 \cdot(1+z)^{2}}-\frac{1}{2}\right)
$$


Beckwith [6] [7] suggests that at $Z \sim 0.4$, a billion years ago, acceleration of the universe increased, as shown in Figure 1. Figure 1 is, if confirmed a good verification of the $\mathrm{Ng}$ [12] hypothesis, and would be a starting point to investigate the role of gravitons in cosmology. The author notes that Buonnano [13] assumes a much lower range of initial frequencies for relic GW than the author. Beckwith [6] [7] obtained a re-acceleration of the universe result as given in Figure 2. The contribution of a low rest mass for 4 dimensional gravitons, as given in Equation (7) leads to a speed-up of acceleration of the expansion of the universe a billion years ago, i.e. for a red shift slightly smaller than 0.5 . Figure 2 below is predicated upon a small 4 dimensional rest mass (stated in Equation (7) for a graviton behaving the same as dark energy)... We will state in our discussions section as to what is needed to give experimental confirmation as to what

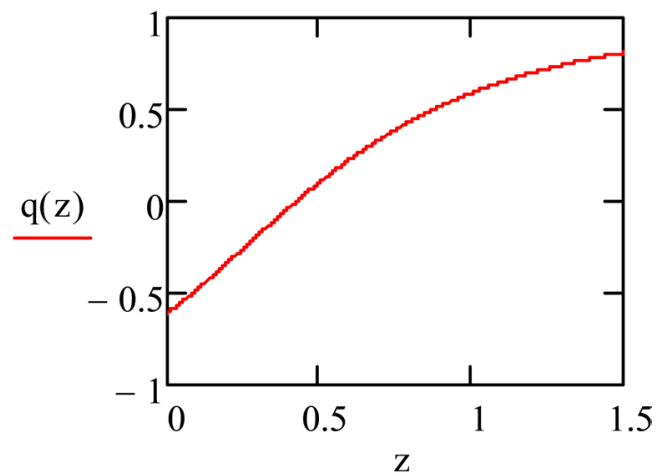

Figure 1. Re-acceleration of the universe based on Beckwith [6]; (note that $q(z)<0$ if $z<$ $0.423)$.

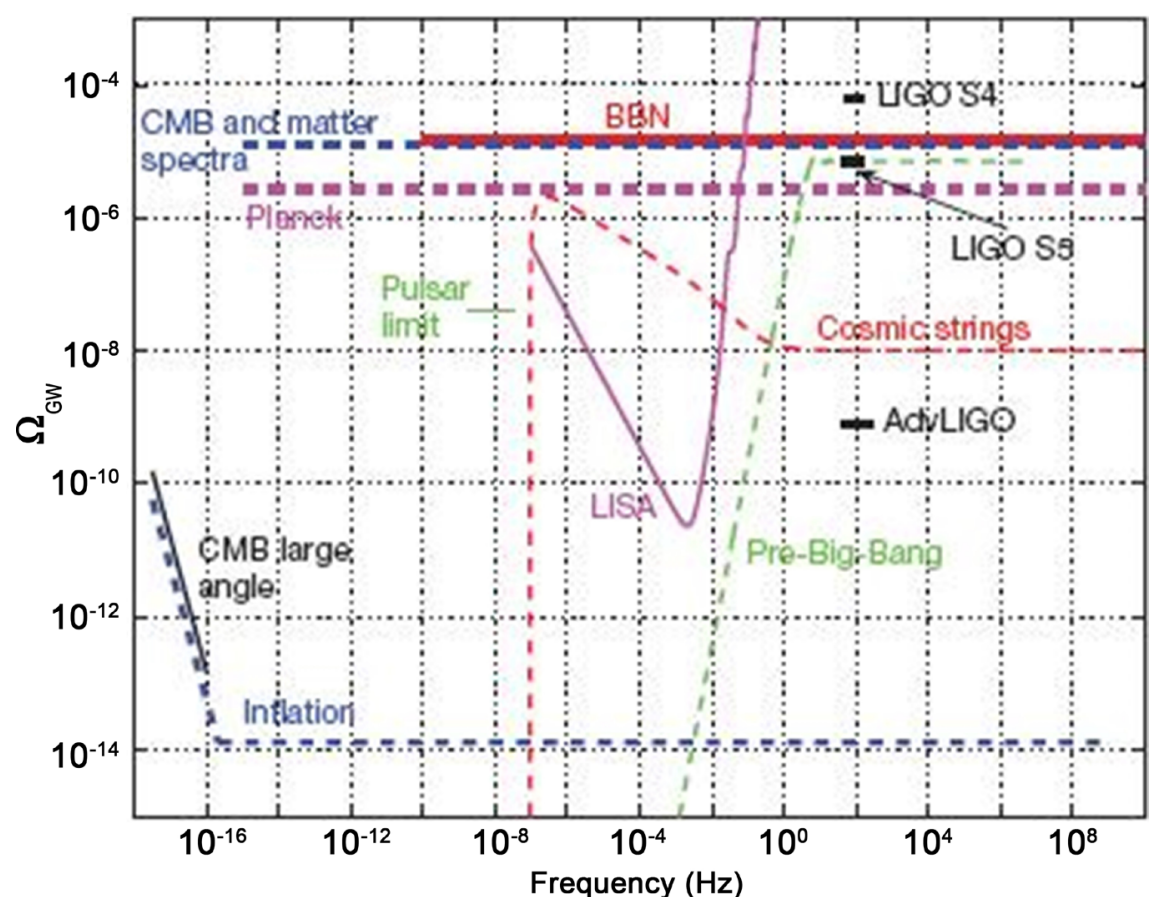

Figure 2. Abbott et al. [14] (2009) shows the relation between GW frequency and GW energy density for different cosmological models. 
is a current for a "massive" graviton which is appropriate for explaining in part, Figure 1.

\section{4. $3^{\text {rd }}$ Part, Reconciling Both Part 1 and Part 2. i.e. What We Are Considering Is a Bridge between Part One and Part 2, i.e. How Can We Reconcile Both of These Regimes?}

\section{From [3] consider the following namely}

Chaotic inflation would be using the approximation that

$$
V(\varphi) \approx \frac{k^{2}}{a^{2}} \cdot \varphi^{2}
$$

Use the approximation that the time derivative is $\mathrm{d} / \mathrm{d} \tau$, and $\varphi \equiv \varphi_{k}$, and if so, then

$$
\begin{aligned}
& \frac{\dot{\varphi}_{k}^{2}}{2}=\left(\frac{3}{8 \pi G} \cdot \frac{\kappa}{a^{2}}+\frac{k^{2}}{a^{2}} \cdot \varphi_{k}^{2}-\rho\right) \\
& \& \\
& \frac{\dot{\varphi}_{k}^{2}}{2}=\left(\frac{3}{8 \pi G} \cdot \frac{\kappa}{a^{2}}+\frac{k^{2}}{a^{2}} \cdot \varphi_{k}^{2}-\frac{16}{3} \cdot c_{1} \cdot B^{4}\right)
\end{aligned}
$$

The last line of Equation (11) states that, if we apply it to the Pre Planckian to Planckian regime, that there will be a change in the energy, which we will call

$$
\Delta E \approx\left(\frac{3}{8 \pi G} \cdot \frac{\kappa}{a^{2}}+\frac{k^{2}}{a^{2}} \cdot \varphi_{k}^{2}-\frac{16}{3} \cdot c_{1} \cdot B^{4}\right)
$$

We then will call this shift in energy, as equivalent to a change in KINETIC energy, and then reference the Virial theorem which in a general form, will be interpreted as

$$
\langle\psi| \text { Kinetic Energy }|\psi\rangle=\langle\psi| r \cdot \nabla V(\text { Potential energy })|\psi\rangle
$$

Leading to

$$
\begin{aligned}
& \langle\psi|\left[\text { Kinetic Energy } \approx\left(\frac{3}{8 \pi G} \cdot \frac{\kappa}{a^{2}}+\frac{k^{2}}{a^{2}} \cdot \varphi_{k}^{2}-\frac{16}{3} \cdot c_{1} \cdot B^{4}\right)\right]|\psi\rangle \\
& \approx\langle\psi|\left(r \cdot \nabla\left[V(\text { Potential energy }) \approx c_{2} / r^{\alpha}\right]\right)|\psi\rangle
\end{aligned}
$$

In the Pre Planckian to Planckian space time, we will approximate, in the instant before time is initialized, formally, the mean value theorem as to the computed values of both the Left and right hand sides of Equation (13) and Equation (14) with the results that we obtain

$$
\begin{aligned}
& \left(\frac{3}{8 \pi G} \cdot \frac{\kappa}{a^{2}}+\frac{k^{2}}{a^{2}} \cdot \varphi_{k}^{2}-\frac{16}{3} \cdot c_{1} \cdot B^{4}\right) \approx-\alpha / r^{\alpha} \equiv-\alpha /(\text { Planck length })^{\alpha} \\
& \Leftrightarrow\left[\alpha /(\text { Planck length })^{\alpha}\right] \approx \frac{16}{3} \cdot c_{1} \cdot B^{4}-\left(\frac{3}{8 \pi G} \cdot \frac{\kappa}{a^{2}}+\frac{k^{2}}{a^{2}} \cdot \varphi_{k}^{2}\right)
\end{aligned}
$$

This variant of dark energy should be compared with the main scheme which has re acceleration of the Universe as its main constituent point. I.e what is 
equivalent to $\mathrm{DE}$ is given below.

This shows in part that $\alpha$ is no longer strictly real valued but is strongly influence by the input from $\varphi_{k}$, i.e. which has real and imaginary components

\section{Conclusions}

To put it mildly, we are concerned as to making a bridge between the initial and second physics realms as to graviton physics and cosmology. In a nutshell, we are proposing a modification of the $1 / \mathrm{r}$ gravitational potential for gravitational waves which in themselves will have gravitons, which is a way to not only get at the DE puzzle, but to answer specifics as to the interaction of gravitons with gravitational waves. i.e. if the $2^{\text {nd }}$ part of our presentation is accurate, we are setting the grounds for examining quantization of gravity, if we confirm both part 2 and part 3 of our inquiry. The gravitational field $g_{u v}$ also has field equations, sort of like those of electromagnetism (the gravitational equations are much more complicated, but they're morally pretty similar). When we quantize the gravitational field as an effective field theory, we find that it too comes in set "quanta," called gravitons. In short, we argue that to come up with a graviton based model of $\mathrm{DE}$ with reacceleration of the universe, and to have it commensurate with the modification of the $1 / \mathrm{r}$ potential, we are really coming up with a program of finding out if gravity can be quantized. In addition what we have done is also to specify a relic black hole genesis for early universe gravitons, which compliments the picture of turbulence in the electroweak era which in turn is relatable to the question of if micro black holes, could contribute to cosmology. This in turn means comparison with [4] and keeping in mind the following, i.e. the diagram given by Abbott et al. [14] (2009) which shows the relation between GW frequency and GW energy density for different cosmological models

\section{A Development to Look into, to Avoid Incompatible Models}

At first glance, this looks hopeless. i.e. the models are incommensurate with each other and we do have a huge problem. A way of having reconciliation may be to consider what is brought up on pages 114 to 115 of Li, Li, Wang, and Wang, [15] as we have, then an examination of the equation of state for DE, that is commensurate with re acceleration of the universe as reading,

$$
\omega_{\text {equation of state DE }}=\frac{\dot{\phi}^{2}-2 V(\phi)}{\dot{\phi}^{2}+2 V(\phi)} \approx \frac{\dot{\phi}^{2}-2 V_{0} \exp \left(-\sqrt{\frac{2}{\tilde{m}}} \frac{\phi}{m_{\text {Plank }}}\right)}{\dot{\phi}^{2}+2 V_{0} \exp \left(-\sqrt{\frac{2}{\tilde{m}}} \frac{\phi}{m_{\text {Plank }}}\right)}
$$

for acceleration of universe $\tilde{m}>1$

Again, this looks like a very hard problem, but in a nutshell, what we need to accomplish is having the following identification made, which may allow for us to make a concrete bridge between formalisms which otherwise look like they have no linkage to each other, i.e. do the following, namely is there a way to link $\phi$ of Equation (16) with $\varphi_{k}$ of Equation (11) and Equation (12) so as to come 
up with an acceptable form of $\phi$ which would satisfy the following equation, given in Equation (18) below, while keeping in mind that [15] gives the following form for a "scalar field" based potential for an accelerating universe, which is called in [15], Equation (17) immediately below this paragraph

Here, we need for acceleration of universe $\tilde{m}>1$

$$
V(\phi)=V_{0} \exp \left(-\sqrt{\frac{2}{\tilde{m}}} \frac{\phi}{m_{\text {Plank }}}\right)
$$

Then, we should try to reconcile the following, if is there a way to link $\phi$ of Equation (16) with $\varphi_{k}$ of Equation (11) and Equation (12)

$$
\omega_{\text {equation of state DE }}=\frac{\dot{\phi}^{2}-2 V(\phi)}{\dot{\phi}^{2}+2 V(\phi)} \approx \frac{\dot{\phi}^{2}-2 V_{0} \exp \left(-\sqrt{\frac{2}{\tilde{m}}} \frac{\phi}{m_{\text {Plank }}}\right)}{\dot{\phi}^{2}+2 V_{0} \exp \left(-\sqrt{\frac{2}{\tilde{m}}} \frac{\phi}{m_{\text {Plank }}}\right)}
$$

for acceleration of universe $\tilde{m}>1$

reconcile above with

$$
\begin{aligned}
& \left(\frac{3}{8 \pi G} \cdot \frac{\kappa}{a^{2}}+\frac{k^{2}}{a^{2}} \cdot \varphi_{k}^{2}-\frac{16}{3} \cdot c_{1} \cdot B^{4}\right) \approx-\alpha / r^{\alpha} \equiv-\alpha /(\text { Planck length })^{\alpha} \\
& \Leftrightarrow\left[\alpha /(\text { Planck length })^{\alpha}\right] \approx \frac{16}{3} \cdot c_{1} \cdot B^{4}-\left(\frac{3}{8 \pi G} \cdot \frac{\kappa}{a^{2}}+\frac{k^{2}}{a^{2}} \cdot \varphi_{k}^{2}\right)
\end{aligned}
$$

In doing so, we would, after a great deal of work perhaps be able to make linkage to the scale factor $a$ which shows up in Equation (15) above. i.e. having results in terms of the scale factor, and $\phi$ while making full identification with the Equation (18) above, may be a way to reformulate the linkage between early $\mathrm{DE}$, which we want. In addition, such restraints upon scale factor a may also begin to explain some of the scale factor behavior which may be implied by Equation (7) and Equation (8) above. Also, if we do this, the final equation of state constraint we have to keep in mind is also given in [15] as

$$
-2 \cdot\left(m_{\text {Plank }}\right)^{2} \cdot \dot{H}=\dot{\phi}^{2}
$$

This should be compared against Equation (7) and justified, if possible, as part of the bridge between early universe expressions of $\mathrm{DE}$ and the re acceleration of the universe.

It is important to note that LIGO as noted in references [16] [17] [18] has already confirmed the basis of a start to gravitational science, which if massive gravitons are used, i.e. then we may be able to give additional details confirming Equation (19) above as well as Equation (11) through Equation (15) above. i.e. $\mathrm{DE}$ if it is commensurate with gravitons then interferometry will add much to being able to give details of the bridge, as represented by Equation (19).

Furthermore, Corda in [19] gave important clues as how to search for the foundations of gravity, itself. i.e. this inquiry should avail itself of the details provided in [19] as well as review the data set research given in [16] [17] [18]. 


\section{Fund}

This work is supported in part by National Nature Science Foundation of China grant No. 11375279.

\section{Conflicts of Interest}

The author declares no conflicts of interest regarding the publication of this paper.

\section{References}

[1] Beckwith, A. Using "Enhanced Quantization” to Bound the Cosmological Constant, and Computing Quantum Number N for Production of 100 Relic Mini Black Holes in a Spherical Region of Emergent Space-Time. http://vixra.org/abs/1805.0378

[2] Beckwith, A. Identifying a Kaluza Klein Treatment of a Graviton Permitting a Deceleration Parameter $\mathrm{Q}(\mathrm{Z})$ as an Alternative to Standard DE.

https://www.researchgate.net/publication/236335939_Identifying_a_Kaluza_Klein_Trea tment_of_a_Graviton_Permitting_a_Deceleration_Parameter_QZ_As_An_Alternative to_Standard_DE

[3] Beckwith, A. Structure Formation in the Early Universe as a Result of Non Linear Electrodynamics Influencing Scale Factor Size with Attendant Changes in Gravitational Potential and Its Relationship to the 3 Body Problem.

http://vixra.org/abs/1805.0357

[4] Inomata, K., Kawasaki, M., Mukaida, K. and Yanagida, T.T. (2018) Double Inflation as a Single Origin of Primordial Black Holes for All Dark Matter and LIGO Observations. Physical Review D, 97, 043514. https://arxiv.org/abs/1711.06129 https://doi.org/10.1103/PhysRevD.97.043514

[5] Corda, C. (2018) Space-Time Can Be Neither Discrete nor Continuous. Modern Physics Letters A, 33, 1850069. https://arxiv.org/abs/1804.03545 https://doi.org/10.1142/S0217732318500694

[6] Beckwith, A.W. (2010) Applications of Euclidian Snyder Geometry to the Foundations of Space Time Physics. EJTP, 7, 241-266. http://vixra.org/abs/0912.0012

[7] Beckwith, A.W. Energy Content of Gravitation as a Way to Quantify Both Entropy and Information Generation in the Early Universe. 58-61.

https://doi.org/10.4236/jmp.2011.22010

[8] Rubakov, V.A. (2002) Classical Theory of Gauge Fields. Princeton University Press, NJ.

[9] Dubovsky, S., et al. (2009) Signatures of a Graviton Mass in the Cosmic Microwave Background. Report UTTG-06-09, TCC-23-09, Physical Review D, 81, 023523. http://arxiv.org/abs/0907.1658

[10] Maartens, R. (2005) Brane World Cosmology. The Physics of the Early Universe, Papantronopoulos, Lect. Notes in Phys., Vol. 653, Springer Verlag, 213-247.

[11] Alves, E., Miranda, O. and de Araujo, J. Can Massive Gravitons Be an Alternative to Dark Energy? http://arxiv.org/pdf/0907.5190

[12] Ng, Y.J. (2008) Spacetime Foam: From Entropy and Holography to Infinite Statistics and Nonlocality. Entropy, 10, 441-461. https://doi.org/10.3390/e10040441

[13] Buonanno, A. (2007) Gravitational Waves. Particle Physics and Cosmology, the Fabric of Space-Time, Elsevier, Oxford, 10-52.

[14] Abbott, B.P., et al. (2009) The LIGO Scientific Collaboration \& The Virgo Collabo- 
ration. An Upper Limit on the Stochastic Gravitational-Wave Background of Cosmological Origin. The LIGO Scientific Collaboration \& The Virgo Collaboration. Nature, 460, 991-993.

[15] Li, M., Li, X.D., Wang, S. and Wang, Y. (2015) Dark Energy. Peking University World Scientific Advance Physics Series, Volume 1, World Scientific, Hackensack.

[16] Abbott, B. P., et al. (2016) LIGO Scientific Collaboration and Virgo Collaboration. Observation of Gravitational Waves from a Binary Black Hole Merger. Physical Review Letters, 116, 061102.

[17] Abbott, B. P., et al. (2016 LIGO Scientific Collaboration and Virgo Collaboration. GW151226: Observation of Gravitational Waves from a 22-Solar-Mass Binary Black Hole Coalescence. Physical Review Letters, 116, 241103.

[18] Abbott, B. P., et al. (2017) LIGO Scientific Collaboration and Virgo Collaboration. GW170104: Observation of a 50-Solar-Mass Binary Black Hole Coalescence at Redshift 0.2. Physical Review Letters, 118, 221101.

[19] Corda, C. (2009) Interferometric Detection of Gravitational Waves: The Definitive Test for General Relativity. International Journal of Modern Physics, D18, 2275-2282. https://arxiv.org/abs/0905.2502 https://doi.org/10.1142/S0218271809015904 